\title{
AN ELM FOR BI-CLASSIFICATION OF VERTICALLY BUNDLED ELECTRICITY MARKET PRICES
}

\author{
S. Anbazhagan ${ }^{1}$ and V. Sivakumar ${ }^{2}$ \\ ${ }^{I}$ Department of Electrical Engineering, Faculty of Engineering and Technology, Annamalai University, India \\ ${ }^{2}$ Anubavam Technologies Private Limited, USA
}

\begin{abstract}
Electricity price forecasting is a challenging problem owing to the very great volatility of price which depends on many factors. This is especially prominent for both producers and consumers where a versatile price forecasting is crucial. This paper contributes an extreme learning machine (ELM) to classify the prices. These price classifications are essential since all market players do not know the precise value of future prices in their deciding procedure. In this paper, bi-classification model is proposed for prices utilizing the pre-specified price threshold. Three alternative classification models based on neural networks (NNs) are also proposed in bi-classification of prices. The performance of the proposed models is compared in terms of classification error and accuracy. The simulation results show that the ELM classification model is superior compared to three other classification models based on NNs. The performances of our models are evaluated using real data from vertically unbundled mainland Spain power system market.
\end{abstract}

Keywords:

Electricity Price Classification, Extreme Learning Machines (ELM), Power System Market, Price Forecasting

\section{INTRODUCTION}

The competitive power system market is broken into three distinct businesses (generation, transmission and distribution) to provide competition in generation and distribution businesses. After vertically unbundling and creating competition, the regulated cost based generation becomes unregulated price-based generation. The monopolistic behavior of the electricity industry will become competitive [1].

In a competitive electricity market, producers and consumers submit bids to the market operator consisting in energy blocks and their corresponding prices. As the end result of a process, hourly energy prices are computed using an appropriate market clearing procedure and accepted selling and buying bids. Both market participants use day-ahead price forecasts to derive their respective bidding strategies to the electricity market. Therefore, accurate price forecasts are crucial for both producers and consumers. Forecasting electricity prices is difficult because unlike demand series, price series present such characteristics as highly unstable, suffering from abnormally low or high price spikes and severe price volatility [2].

In a competitive electricity market, more and more attention has been paid to price forecasting compared to load forecasting. However, those procedures are either too complex to implement or too simple to have enough accuracy [3]. Artificial neural network (ANN) technique is a simple and powerful tool for forecasting [4][7]. Hybrid intelligent methods are complex to implement and also too powerful tool for forecasting [8]-[15]. However, the accuracy is not satisfying.

Although electricity is a different type of commodity that cannot be stored in bulk quantities and is also related to interconnected grid management. These problems highlight severe price volatility in the competitive electricity market. For instance, electricity price depends on its previous values (historical values) and load values [17]. They are evidently indicated that there is a strong require in the electric power business for convincingly accurate tools that properly forecast electricity prices.

The existing approaches try to forecast the precise value of prices at next hours by estimating the true underlying price formation procedure. However, not all market players know the accurate value of future prices in their deciding procedure. An example of threshold-based decision can be found in users with on-site generation facilities. These facilities only purchase electricity from the grid if the prices are below the marginal cost of operating the on-site generation equipment. In these types of applications where the accurate value of prices is not required, the prediction can be reduced to classification sub problems in which the class of future prices is of interest [16].

Price classification [16], [21]-[24] has become a crucial research in electrical engineering in current years. Among the three alternative classification models based on neural networks (NNs), application of extreme learning machines (ELM) has been contributed in this paper, because of generalized single hidden layer feed forward networks (SLFNs), that performs well in both regression and classification applications, which is usually hard to model with conventional models.

This paper proposes ELM approach to classify next-week prices in the Spanish market. The electricity price bi-classification procedure is as an alternative to price prediction. An electricity prices are classified based on the pre-specified thresholds. These thresholds are specified by the market players based on their needs. The main contribution of this manuscript is proposing a electricity price bi-classification that could be realized using ELM.

This paper is organized as follows. Section 2 presents data source and various classification models for bi-classification of electricity prices. Section 3 presents the numerical results of various bi-classification models from the Spanish market. Finally, the conclusions are given in section 4 .

\section{METHODOLOGY}

This section describes the data source and three classification models based on NNs such as feed forward NN (FFNN), probabilistic NN (PNN), and learning vector quantization (LVQ) and ELM classification models for bi-classification of day-ahead 
Spanish electricity market. There are 16 input features for the proposed binary classification models. The goals of the binary classification models are to classify each pattern as belonging, or not belonging, to a particular class. Belonging is signified by the output unit giving a response of 1 , not belonging is indicted by a response of -1 .

\subsection{DATA SOURCE}

The Spanish electricity market data source [18] and the suitable selection of input features are discussed in [21]-[23]. Biclassification thresholds are considered for the Spanish market for the year 2002: $T_{1}=0, T_{2}=37$ and $T_{3}=158$ with all in euro per megawatt hour. $T_{1}, T_{2}$ and $T_{3}$ are the price floors, annual average and price cap of the prices. Normally, the users may set their price thresholds based on their own operating criteria. From these price thresholds, the bi-class distribution is

- Class 1: (Prices between $T_{1}$ and $T_{2}$ )

- Class 2: (Prices between $T_{2}$ and $T_{3}$ )

\subsection{BI-CLASSIFICATION OF ELECTRICITY PRICES USING FFNN}

In the first model of this paper, the FFNN is selected as NN type with Levenberg-Marquardt (LM) training. The FFNN classification model with one hidden layer is used for biclassification of electricity prices is shown in Fig.1. The hidden layer neurons use non-linear hyperbolic-tangent-sigmoid and the output layer neuron use pure linear activation functions. Equations used in the FFNN bi-classification model with only one hidden layer are discussed in [19] [23].

\subsection{BI-CLASSIFICATION OF ELECTRICITY PRICES USING PNN}

The second model of this paper, PNN was devised by Speckt in 1990. The PNN structure consisting of an input layer, a single hidden layer (radial basis layer), and an output layer (competitive layer) are as shown in Fig.2. Equations that are used in the NN model are shown in Eq.(1) - Eq.(5).

$$
\begin{gathered}
X_{j}^{r}=\varphi\left(\left\|\vec{f}-\vec{c}_{j}\right\| * b^{i r}\right) \\
\varphi(x)=\exp \left(-x^{2}\right) \\
b^{i r}=0.8326 / s \\
S_{i}=\sum_{j=1}^{h} W_{j i}^{r c} * X_{j}^{r} \\
Y_{i}=\left\{\begin{array}{lc}
1 & \text { if } S_{i} \text { is } \max \text { of }\left\{S_{1}, S_{2}\right\} \\
0 & \text { else }
\end{array}\right.
\end{gathered}
$$

where, $i=1,2$, and $j=1,2, \ldots, h$ (number of hidden neurons), $W^{r c}$ are the competitive layer weights and $Y_{i}$ is the $i^{\text {th }}$ bi-classification of electricity price outputs.

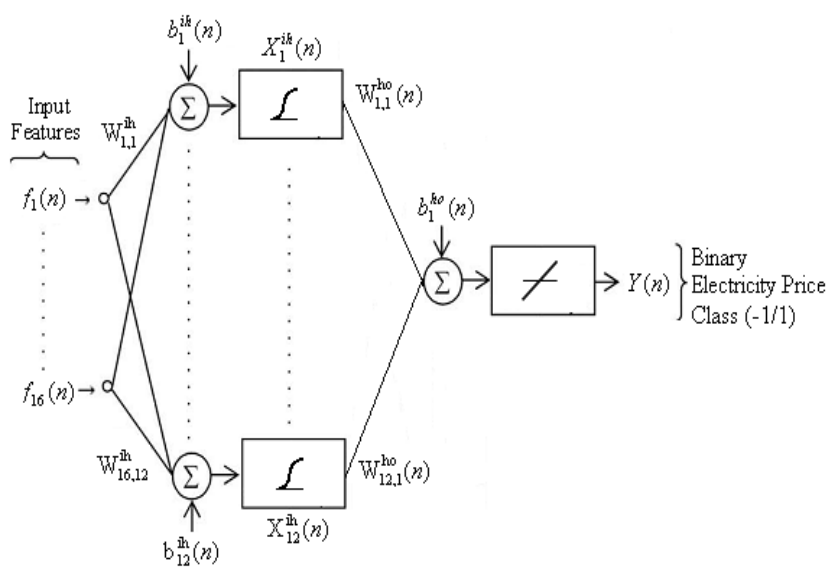

Fig.1. Implementation of FFNN for bi-classification of electricity prices

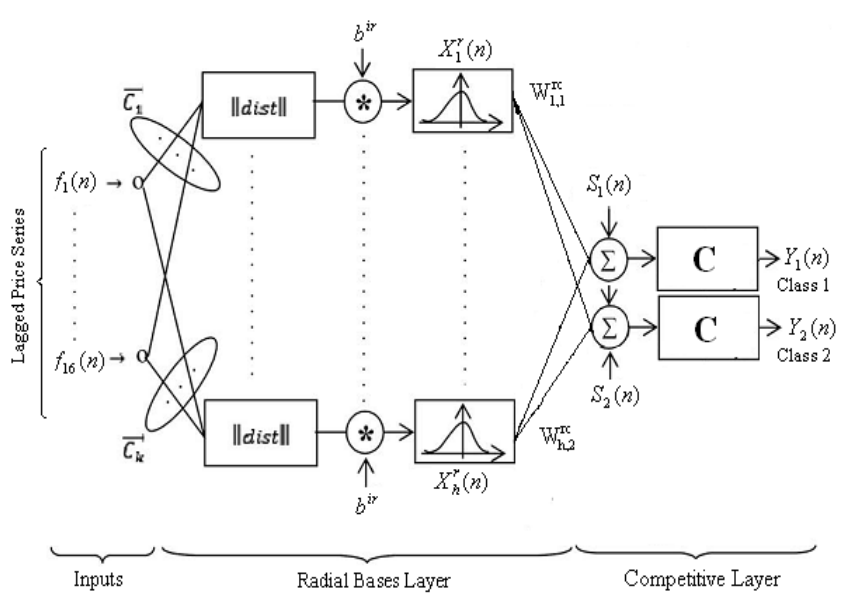

Fig.2. Implementation of PNN for bi-classification of electricity prices

\subsection{BI-CLASSIFICATION OF ELECTRICITY PRICES USING LVQ}

The third model of this paper, LVQ was devised by Kohonen in 1990. The LVQ structure structures consisting of an input layer, a single hidden layer (competitive layer), and an output layer (linear layer) are as shown in Fig.3. Equations that are used in the NN model are shown in Eq.(6) - Eq.(8).

$$
\begin{gathered}
\mathrm{S}_{\mathrm{j}}=\left\|\vec{f}-\vec{c}_{j}\right\| \\
X_{i}^{c}=\left\{\begin{array}{c}
1 \text { if } S_{i} \text { is } \max \text { of }\left\{S_{1}, \ldots \ldots, S_{h}\right\} \\
0 \quad \text { else }
\end{array}\right. \\
Y_{i}=\sum_{j=1}^{h} W_{j i}^{c o} * X_{j}
\end{gathered}
$$

where, $i=1,2$, and $j=1,2, \ldots, h, X_{j}^{c}$ value is the $j^{\text {th }}$ output of competitive layer and $W^{c o}(n)$ are the weights from the competitive layer to the output layer. 


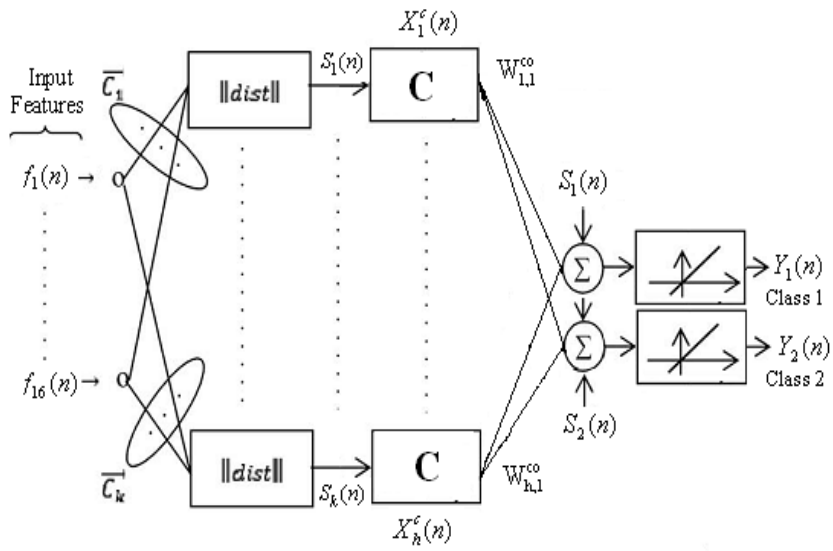

Fig.3. Implementation of LVQ for bi-classification of electricity prices

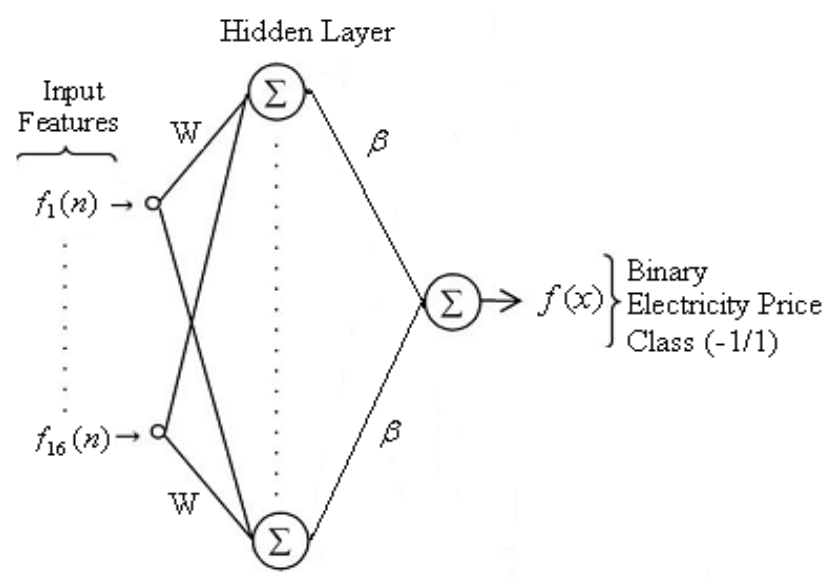

Fig.4. Implementation of ELM for bi-classification of electricity prices

\subsection{BI-CLASSIFICATION OF ELECTRICITY PRICES USING ELM}

The fourth model of this paper, the ELM is a generalized SLFN. It has a structures consisting of an input layer, a single hidden layer, and an output layer as shown in Fig.4. Unlike these conventional implementations, a new learning algorithm called ELM which arbitrarily gives all the hidden nodes parameters of generalized SLFNs and analytically learns the output weights of SLFNs. The target functions or the training datasets are not influence the hidden node parameters. An ELM algorithm can determine all the parameters are determined analytically instead of being tuned. In theory, this algorithm tends to provide the good generalization performance at extremely fast learning speed. In conventional learning theory and implementations, one has to see the training data before generating the hidden node parameters [20]. In ELM learning theory and implementations, one can generate the hidden node parameters before seeing the training data. Equations that are used in the ELM model are shown in Eq.(9) and Eq.(10).

$$
H \beta=T
$$

The determination of the output weights between the hidden layer and the output layer is to find the least-square solution to the given linear system [25]. The minimum norm least-square (LS) solution to the linear system Eq.(9) is

$$
f(x)=\sum_{i=1}^{l} \beta_{i} h_{i}(x),
$$

where, $H$ is the hidden-layer output matrix, $\beta$ is the matrix of output weights and $T$ is the matrix of targets. $h_{i}(x)$ is the output of the $i^{\text {th }}$ hidden node.

\subsection{IMPLEMENTATION PROCEDURE OF PROPOSED CLASSIFICATION MODELS}

The proposed models classification procedure is shown in Fig.5. The elaborated statement of procedure is given in [23].

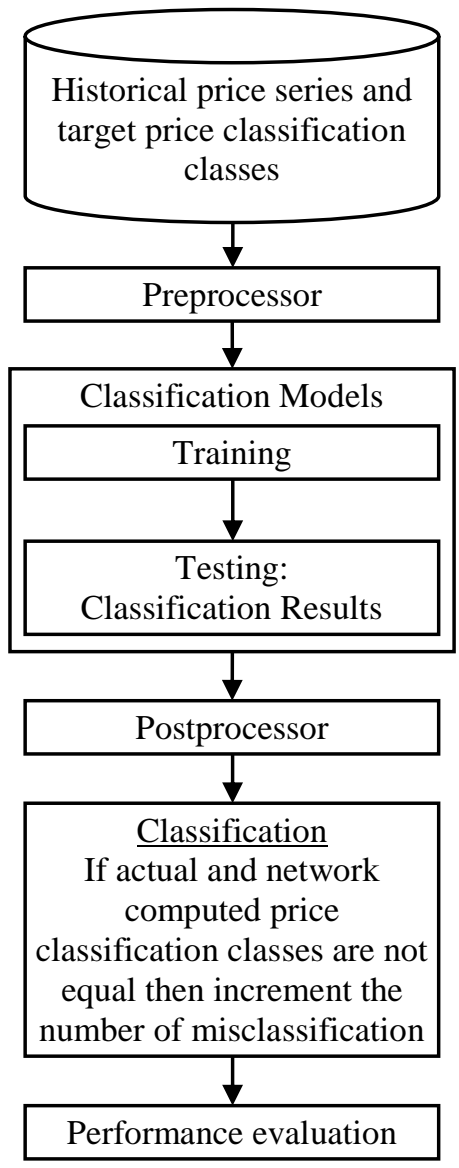

Fig.5. Classification procedure of proposed classification models

The performance of the trained bi-classification model is then evaluated by mean percentage classification error (MPCE) and percentage classification accuracy (PCA). The MPCE can be defined as,

$$
M P C E=\left(\frac{N_{m c}}{N_{t o t}}\right) \times 100
$$

The PCA is given by,

$$
P C A=\left(\frac{N_{t o t}-N_{m c}}{N_{t o t}}\right) \times 100
$$

where, $N_{m c}$ and $N_{t o t}$ are the number of misclassified and total number of classified hours respectively. The simulations were 
carried out in AMD processor with $2 \mathrm{GHz}$ and $1 \mathrm{~GB}$ RAM. The simulation was conducted in all proposed bi-classification models.

\section{NUMERICAL RESULTS}

This section describes the case study of mainland Spain market for the year 2002 by proposed bi-classification models.

\subsection{CASE STUDY}

The methodology described above has been applied to biclassify the electricity prices of mainland Spain market. The case study about the training and testing (bi-classification) weeks of mainland Spain market was discussed in [5], [21]-[23]. The Data set composition for Spanish electricity market in the year 2002 are given in Table.1. Given the New York, CAPITAL zone dataset consists of locational-based marginal price in the year 2010 are investigated and the consideration in this work, presenting other more detailed error/accuracy measures, such as a MPCE and PCA, is not possible due to page limitations.

Table.1. Data set composition for the Spanish electricity market in the year 2002

\begin{tabular}{|c|c|c|c|c|}
\hline $\begin{array}{c}\text { Classification } \\
\text { Weeks }\end{array}$ & Application & $\begin{array}{c}\text { Below } \\
\text { Average }\end{array}$ & $\begin{array}{c}\text { Above } \\
\text { Average }\end{array}$ & Total \\
\hline \multirow{2}{*}{ Winter } & Training Vector & 259 & 749 & 1008 \\
\cline { 2 - 5 } & Testing Vector & 65 & 103 & 168 \\
\hline \multirow{2}{*}{ Spring } & Training Vector & 366 & 642 & 1008 \\
\cline { 2 - 5 } & Testing Vector & 62 & 106 & 168 \\
\hline \multirow{2}{*}{ Summer } & Training Vector & 491 & 517 & 1008 \\
\cline { 2 - 5 } & Testing Vector & 110 & 58 & 168 \\
\hline \multirow{2}{*}{ Fall } & Training Vector & 605 & 403 & 1008 \\
\cline { 2 - 5 } & Testing Vector & 130 & 38 & 168 \\
\hline
\end{tabular}

\subsection{PRICE CLASSIFICATION WITH PROPOSED BI-CLASSIFICATION MODELS}

In the bi-classification models, training method and spread factor were determined using trial and error approach. Several attempts were made until the proper number of hidden layers, number of neurons in hidden layer, spread factor and different activation functions were reached. The classification models selected after these attempts produced minimal error in both training and testing.

The proposed classification models have input layer composed of sixteen neurons and output layer with one neuron (bi-classification of electricity prices) in FFNN and ELM; two neurons in PNN and LVQ classification models. The three NN classification models are implemented using the MATLAB NN toolbox. In ELM classification model, elm.m freely available MATLAB code was used for training and testing the model [20]. The size of the input pattern is 16 (historical price series) $\times 1008$ (42 days training period $\times 24$ hours), and the size of the target pattern is 1 or 2 (bi-classification of prices) $\times 1008$ in this proposed classification models.

\subsubsection{Price Classification with NN Models:}

In NN classification models, the resultant number of neurons in the hidden layer for FFNN, LVQ and ELM classification models produced minimal MPCE error and maximal PCA in both training and testing in each of the considered weeks and are shown in Table.2. The resultant spread factor for each of the considered weeks for PNN classification model produced minimal MPCE error, maximal PCA in both training and testing and are shown in Table.3.

Table.2. Best number of neurons in the hidden layer obtained with the FFNN, LVQ and ELM classification models on mainland Spain market in the year 2002

\begin{tabular}{|c|c|c|}
\hline $\begin{array}{c}\text { Classification } \\
\text { Models }\end{array}$ & $\begin{array}{c}\text { Classification } \\
\text { Weeks }\end{array}$ & $\begin{array}{c}\text { Number of Neurons } \\
\text { in the Hidden Layer }\end{array}$ \\
\hline \multirow{4}{*}{ FFNN } & Winter & 12 \\
\cline { 2 - 3 } & Spring & 12 \\
\cline { 2 - 3 } & Summer & 12 \\
\cline { 2 - 3 } & Fall & 12 \\
\hline \multirow{4}{*}{ LVQ } & Winter & 10 \\
\cline { 2 - 3 } & Spring & 10 \\
\cline { 2 - 3 } & Summer & 10 \\
\cline { 2 - 3 } & Fall & 10 \\
\hline \multirow{4}{*}{ ELM } & Winter & 24 \\
\cline { 2 - 3 } & Spring & 19 \\
\cline { 2 - 3 } & Summer & 15 \\
\cline { 2 - 3 } & Fall & 18 \\
\hline
\end{tabular}

Table.3. Best spread factor obtained with the NN classification models on mainland Spain market in year 2002

\begin{tabular}{|c|c|c|}
\hline $\begin{array}{c}\text { Neural Network } \\
\text { Classification Models }\end{array}$ & $\begin{array}{c}\text { Classification } \\
\text { Weeks }\end{array}$ & $\begin{array}{c}\text { Spread } \\
\text { Factor }\end{array}$ \\
\hline \multirow{3}{*}{ PNN } & Winter & 0.10 \\
\cline { 2 - 3 } & Spring & 0.40 \\
\cline { 2 - 3 } & Summer & 0.30 \\
\cline { 2 - 3 } & Fall & 0.30 \\
\hline
\end{tabular}

The Table.4 shows how the MPCE and PCA vary in accordance with bi-classification of electricity prices considered in each of the considered weeks obtained with the NN classification models on mainland Spain market for the year 2002. It can be observed from Table.4, the FFNN has improved most of the MPCE and PCA average values compared with other NN classification models. Finally, minimal MPCE and maximal PCA for the Spanish electricity market has an average value of $4.2 \%$ and $95.83 \%$ obtained using FFNN classification model.

\subsubsection{Price Classification with ELM Model:}

In ELM classification model, different activation functions are supported. For bi-classification of electricity prices, it runs several times to evaluate different number of hidden neurons in the model. Fig. 6 shows the different number of hidden neurons versus the training accuracy by ELM for the Spanish summer week for binary electricity price classification. The performance of ELM increases for the different number of hidden neurons from 5 to 25 is shown in Fig.6. Maximum performance of ELM training 
accuracy 0.93 occurs at the hidden neuron 24. After that the performance of ELM is gradually reducing for Spanish summer week for binary electricity price classification. The resultant number of neurons in the hidden layer for ELM classification model produced minimal MPCE error and maximal PCA in both training and testing in each of the considered weeks are shown in Table.2. Finally, bi-classification of electricity prices is to evaluate different activation functions in the best number of neurons in the hidden layer.

The sigmoidal, sine and hard limit activation functions are used for bi-classification of electricity prices. The performance for each of the considered weeks for ELM classification model on Spanish market that produced MPCE error, PCA in both training and testing for different activation functions are shown in Table.5. It can be observed from Table.5, the sigmoidal activation function has improved most of the MPCE and PCA average values compared with other activation functions. Finally, minimal MPCE and maximal PCA for the Spanish electricity market has an average value of $3.2 \%$ and $96.87 \%$ obtained using sigmoidal activation function.

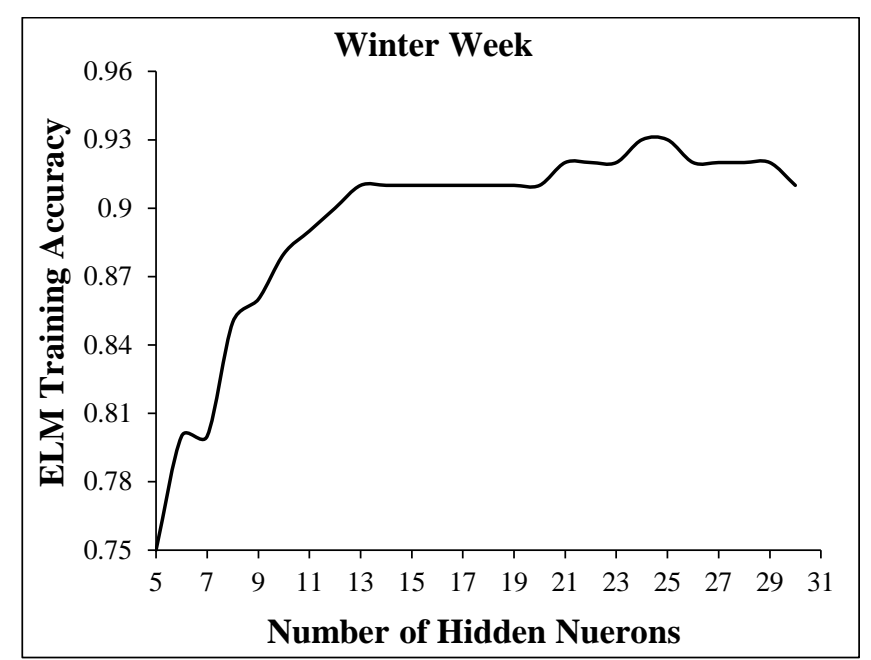

Fig.6. Performance of ELM training accuracy for the Spanish winter week

\subsubsection{Comparison of Proposed Classification Models:}

It is perceived from the available literature that traditional price forecasting approaches are generally developed for numerical prediction or point-forecasting. Binary classification of electricity prices is not proposed in the previous literature. Hence, comparative analysis made for among the classification models proposed. The Table. 4 and Table.5 give statistical analysis for four weeks obtained with the three classification models based on NNs (such as FFNN, PNN, and LVQ) and proposed ELM classification models on mainland Spain market for the year 2002.

The Table. 4 shows the minimum MPCE is $3.0 \%$ and maximum PCA is $97.02 \%$ occurred in spring week of FFNN classification model for the bi-classification of prices when compared with other NN classification models on Spanish market. The Table.5 shows the minimum MPCE is $1.8 \%$ and maximum PCA is $98.21 \%$ occurred in spring week of ELM classification model using sigmoidal activation function for the bi-classification of prices when compared with other activation functions on Spanish market.
Table.4. MPCE and PCA obtained with the proposed NN classification models on mainland Spain market in year 2002

\begin{tabular}{|c|c|c|c|}
\hline \multicolumn{2}{|c|}{ Classification } & \multicolumn{2}{c|}{ Performance Evaluation } \\
\hline NN Models & Weeks & MPCE & PCA \\
\hline \multirow{4}{*}{ FFNN } & Winter & 3.6 & 96.43 \\
\cline { 2 - 4 } & Spring & 3.0 & 97.02 \\
\cline { 2 - 4 } & Summer & 5.4 & 94.64 \\
\cline { 2 - 4 } & Fall & 4.8 & 95.24 \\
\cline { 2 - 4 } & Average, \% & 4.2 & 95.83 \\
\hline \multirow{4}{*}{ PNN } & Winter & 4.2 & 95.83 \\
\cline { 2 - 4 } & Spring & 4.8 & 95.24 \\
\cline { 2 - 4 } & Summer & 12.5 & 87.50 \\
\cline { 2 - 4 } & Fall & 5.4 & 94.64 \\
\cline { 2 - 4 } & Average, \% & 6.7 & 93.30 \\
\hline \multirow{7}{*}{ LVQ } & Winter & 20.2 & 79.76 \\
\cline { 2 - 4 } & Spring & 6.0 & 94.05 \\
\cline { 2 - 4 } & Summer & 17.3 & 82.74 \\
\cline { 2 - 4 } & Fall & 8.3 & 91.67 \\
\cline { 2 - 4 } & Average, \% & 13.0 & 87.06 \\
\hline
\end{tabular}

From the Table.4, it is observed the minimum MPCE and maximum PCA is $4.2 \%$ and $95.83 \%$ respectively occurred on an average in FFNN classification model for bi-classification of prices when compared with other NN classification models for all the four weeks of mainland Spain market in year 2002.

Table.5. MPCE and PCA obtained with the proposed ELM classification model on mainland Spain market in year 2002 for different activation functions

\begin{tabular}{|c|c|c|c|}
\hline \multirow{2}{*}{$\begin{array}{c}\text { ELM } \\
\text { Activation } \\
\text { Functions }\end{array}$} & $\begin{array}{c}\text { Classification } \\
\text { Weeks }\end{array}$ & \multicolumn{2}{|c|}{$\begin{array}{c}\text { Performance } \\
\text { Evaluation }\end{array}$} \\
\cline { 2 - 4 } & & MPCE & PCA \\
\hline \multirow{4}{*}{ Sigmoid } & Winter & 3.0 & 97.02 \\
\cline { 2 - 4 } & Spring & 1.8 & 98.21 \\
\cline { 2 - 4 } & Summer & 4.2 & 95.83 \\
\cline { 2 - 4 } & Fall & 3.6 & 96.43 \\
\cline { 2 - 4 } & Average, \% & 3.2 & 96.87 \\
\hline \multirow{4}{*}{ Sine } & Winter & 3.6 & 96.43 \\
\cline { 2 - 4 } & Spring & 3.6 & 96.43 \\
\cline { 2 - 4 } & Summer & 4.8 & 95.24 \\
\cline { 2 - 4 } & Fall & 3.6 & 96.43 \\
\cline { 2 - 4 } & Average, \% & 3.9 & 96.13 \\
\hline \multirow{4}{*}{ Hard Limit } & Winter & 26.2 & 73.81 \\
\cline { 2 - 4 } & Spring & 11.9 & 88.10 \\
\cline { 2 - 4 } & Summer & 29.8 & 70.24 \\
\cline { 2 - 4 } & Fall & 25.0 & 75.00 \\
\cline { 2 - 4 } & Average, \% & 23.2 & 76.79 \\
\hline
\end{tabular}

From the Table.5, it is observed the minimum MPCE and maximum PCA is $3.2 \%$ and $96.87 \%$ respectively occurred on an average in ELM classification model using sigmoidal activation function for bi-classification prices when compared with other 
activation functions for all the four weeks of mainland Spain market in year 2002 .

Finally, it is observed from the Table. 4 and Table. 5 give minimum MPCE and maximum PCA is $3.2 \%$ and $96.87 \%$ respectively occurred on an average in ELM classification model using sigmoidal activation function is capable of classifying the electricity market prices efficiently. So, we can easily say that ELM classification model possesses better classifying abilities than the three classification models based on $\mathrm{NN}$ and its performance was least affected by the price volatility.

The simulation was conducted in proposed bi-classification models. Moreover, the ELM presents lower modeling complexity: the average computation time is less than $1 \mathrm{~ms}$. In a competitive electricity market, the fast binary classification of prices is also crucial for real-life processing.

\section{CONCLUSION}

The bi-classification of electricity prices are vital because all market participants do not know the exact value of the future prices in their decision making process. Binary price classification results of the three ANN and ELM models on the Spanish electricity market for the four weeks of the year 2002 are reported, yielding an average weekly MPCE and PCA which is close to $3.2 \%$ and $96.87 \%$ for ELM model using sigmoidal activation function, while the average computation time is less than $1 \mathrm{~ms}$ and lower modeling complexity. Hence, it is suitable for real-time competitive electricity market. In a competitive electricity market, the fast classification of prices is also essential for real-life applications. The research work is underway in order to develop better feature selection algorithm for different electricity markets and classification models.

\section{REFERENCES}

[1] S.N. Singh, "Electric Power Generation, Transmission and Distribution", $2^{\text {nd }}$ Edition, Prentice-Hall, 2008.

[2] A.J. Conejo, M.A. Plazas, R. Espinola and A. B. Molina, "Day-Ahead Electricity Price Forecasting using the Wavelet Transform and ARIMA Models", IEEE Transactions on Power Systems, Vol. 20, No. 2, pp. 1035-1042, 2005.

[3] H.Y. Yamin, S.M. Shahidehpour and Z. Li, "Adaptive Short-Term Electricity Price Forecasting using Artificial Neural Networks in the Restructured Power Markets", International Journal of Electrical Power and Energy Systems, Vol. 26, No. 8, pp. 571-581, 2004.

[4] P. Mandal, T. Senjyu and T. Funabashi, "Neural Networks Approach to Forecast Several Hour ahead Electricity Prices and Loads in Deregulated Market", Energy Conversion and Management, Vol. 47, No. 15-16, pp. 2128-2142, 2006.

[5] J.P.S. Catalao, S.J.P.S. Mariano and V.M.F. Mendes, "Short-Term Electricity Prices Forecasting in a Competitive Market: A Neural Network Approach", Electric Power Systems Research, Vol. 77, Vo. 10, pp. 1297-1304, 2007.

[6] R. Gareta, L.M. Romeo and A. Gil, "Forecasting of Electricity Prices with Neural Networks", Energy Conversion and Management, Vol. 47, No. 13-14, pp. 17701778, 2006.
[7] H.T. Pao, "Forecasting Electricity Market Pricing using Artificial Neural Networks", Energy Conversion and Management, Vol. 48, No. 3, pp. 907-912, 2007.

[8] N. Amjady, "Day-Ahead Price Forecasting of Electricity Markets by a New Fuzzy Neural Network", IEEE Transactions on Power Systems, Vol. 21, No. 2, pp. 887896, 2006.

[9] N. Amjady and H. Hemmati, "Day-Ahead Price Forecasting of Electricity Markets by a Hybrid Intelligent System", International Transactions on Electrical Energy Systems, Vol. 19, No. 1, pp. 89-102, 2009.

[10] N.M. Pindoriya, S.N. Singh and S.K. Singh, “An Adaptive Wavelet Neural Network-based Energy Price Forecasting in Electricity Markets", IEEE Transactions on Power Systems, Vol. 23, No. 3, pp. 1423-1432, 2008.

[11] J.P.S. Catalao, H.M.I. Pousinho and V.M.F. Mendes, "Neural Networks and Wavelet Transform for Short-Term Electricity Prices Forecasting", Proceedings of $15^{\text {th }}$ International Conference on Intelligent System Applications to Power Systems, pp. 1-5, 2009.

[12] J.P.S. Catalao, H.M.I. Pousinho and V.M.F. Mendes, "Short-Term Electricity Prices Forecasting in a Competitive Market by a Hybrid Intelligent Approach", Energy Conversion and Management, Vol. 52, No. 2, pp. 10611065, 2011.

[13] M. Shafie-khah, M.P. Moghaddam and M.K. Sheikh-ElEslami, "Price Forecasting of Day-Ahead Electricity Markets using a Hybrid Forecast Method", Energy Conversion and Management, Vol. 52, No. 5, pp. 21652169, 2011.

[14] N. Amjady and F. Keynia, "Day-Ahead Price Forecasting of Electricity Markets by Mutual Information Technique and Cascaded Neuro-Evolutionary Algorithm", IEEE Transactions on Power Systems, Vol. 24, No. 1, pp. 306318, 2009.

[15] J. P.S. Catalao, H.M.I. Pousinho and V.M.F. Mendes, "Hybrid Wavelet-PSO-ANFIS Approach for Short-Term Electricity Prices Forecasting", IEEE Transactions on Power Systems, Vol. 26, No. 1, pp. 137-144, 2011.

[16] H. Zareipour, A. Janjani, H. Leung, A. Motamedi and A. Schellenberg, "Classification of Future Electricity Market Prices”, IEEE Transactions on Power Systems, Vol. 26, No. 1, pp. 165-173, 2011.

[17] N. Amjady and F. Keynia, "Application of a New Hybrid Neuro-Evolutionary System for Day-Ahead Price Forecasting of Electricity Markets", Applied Soft Computing, Vol. 10, No. 3, pp. 784-792, 2010.

[18] Spanish Electricity Market, Available at: www.omel.com, Accessed on 2017.

[19] O. Er, N. Yumusak and F. Temurtas, "Chest Diseases Diagnosis using Artificial Neural Networks", Expert Systems with Applications, Vol. 37, No. 12, pp. 7648-7655, 2010.

[20] Extreme Learning Machines (ELM) and its Applications, Available at: http://www3.ntu.edu.sg/home/egbhuang/, Accessed on 2017.

[21] S. Anbazhagan and N. Kumarappan, "A Neural Network Approach to Day-Ahead Deregulated Electricity Market Prices Classification", Electric Power Systems Research, Vol. 86, pp. 140-150, 2012. 
[22] S. Anbazhagan and N. Kumarappan, "Day-Ahead Deregulated Electricity Market Price Classification using Neural Network Input Featured by DCT", International Journal of Electrical Power and Energy Systems, Vol. 37, No. 1, pp. 103-109, 2012.

[23] S. Anbazhagan and N. Kumarappan, "Binary Classification of Day-Ahead Deregulated Electricity Market Prices using Neural Network Input Featured by DCT”, ICTACT Journal on Soft Computing, Vol. 2, No. 4, pp. 384-390, 2012.
[24] H. Zareipour, A. Janjani, H. Leung, A. Motamedi and A. Schellenberg, "Electricity Price Thresholding and Classification", Proceedings of IEEE Power and Energy Society General Meeting, pp. 1-7, 2011.

[25] W.K. Wong and Z.X. Guo, "A Hybrid Intelligent Model for Medium-Term Sales Forecasting in Fashion Retail Supply Chains using Extreme Learning Machine and Harmony Search Algorithm", International Journal of Production Economics, Vol. 128, No. 2, pp. 614-624, 2010. 